\title{
A Pilot Study of the Effect of Daikenchuto on Rectal Sensation in Patients with Irritable Bowel Syndrome
}

\author{
Andres Acosta, Michael Camilleri, ${ }^{*}$ Sara Linker-Nord, Irene Busciglio, Johanna Iturrino, Lawrence A Szarka, and \\ Alan R Zinsmeister \\ Clinical Enteric Neuroscience Translational and Epidemiological Research (C.E.N.T.E.R.), Mayo Clinic, Rochester, Minnesota, USA
}

\section{Background/Aims}

Daikenchuto (TU 100), a botanical agent that modulates gastrointestinal nerves, is used in the treatment of motility and functional disorders. Our aim was to study the effects of TU-100 on rectal compliance and sensation in patients with irritable bowel syndrome (IBS).

\section{Methods}

In 20 patients per treatment arm, we conducted a single-center, randomized, parallel-group, double-blind, placebo-controlled, singledose pharmacodynamics study evaluating the effects of TU-100, $15 \mathrm{~g}$ ( $5 \mathrm{~g}$ t.i.d. [means 3 times a day]), for $14-16$ consecutive days on rectal compliance and rectal sensation (thresholds and sensation ratings), all measured at baseline and on the last day of medication treatment. The primary endpoint was rectal sensation thresholds and sensation ratings in response to balloon distension at $32 \mathrm{mmHg}$. Secondary endpoints were rectal compliance, sensation thresholds, ratings and tone (fasting and postprandial), bowel pattern, abdominal pain (average and worst severity) and bloating scores, IBS quality of life and safety profile.

\section{Results}

Rectal sensation ratings post-treatment were significantly associated with baseline (pre-treatment) ratings and with level of anxiety or stress recorded at the time of the sensation testing. There were no effects of TU-100 treatment on rectal sensation ratings, sensation thresholds, rectal fasting or postprandial tone, rectal compliance, bowel function, abdominal pain or bloating scores, or IBS quality of life.

\section{Conclusions}

TU-100 did not significantly affect rectal compliance and sensation in patients with IBS in this study.

(J Neurogastroenterol Motil 2016;22:69-77)

Key Words

Abdominal pain; Anxiety; Humans; Quality of life

Received: July 22, 2015 Revised: September 17, 2015 Accepted: September 19, 2015

(a) This is an Open Access article distributed under the terms of the Creative Commons Attribution Non-Commercial License (http://creativecommons. org/licenses/by-nc/4.0) which permits unrestricted non-commercial use, distribution, and reproduction in any medium, provided the original work is properly cited.

*Correspondence: Michael Camilleri, MD

Mayo Clinic, Charlton 8-110, 200 First Street S.W., Rochester, Minnesota 55905, USA

Tel: +1-507-266-2305, E-mail: camilleri.michael@mayo.edu 


\section{Introduction}

Daikenchuto (TU-100) is an herbal medicine that has been used to treat adhesive bowel obstruction and postoperative ileus in Japan. TU-100 treatment may also be useful for the management of radiation-induced enteritis. ${ }^{1}$ TU-100 is a mixture of extract powders from dried Sichuan pepper, processed ginger, ginseng, and maltose powder. Although the exact mechanism of its effect is unknown, it is thought that TU-100 may increase gastrointestinal (GI) motility by an up-regulation of the calcitonin gene related peptide (CGRP), which is a powerful vasoactive substance ${ }^{2}$ and is involved in visceral sensation. ${ }^{3}$

TU-100 also has been demonstrated to stimulate GI motility. In in vivo experiments, the ameliorating effect of TU-100 on laparotomy induced or chemically-induced intestinal dysmotility was inhibited by atropine (a muscarinic receptor antagonist), serotonin type 4 receptor antagonist, or cholecystokinin antagonist. Intraduodenal and intrajejunal administration of the extract powder $(0.5,1.5$, and $3.0 \mathrm{~g}$ doses $)$ increased the motility of the duodenum, proximal jejunum, and distal jejunum in conscious dogs in a dose-dependent manner. Atropine and hexamethonium inhibited the effects of the extract powder at all sites. ${ }^{4,5}$ These results suggest that the effects of TU-100 are mainly mediated by the cholinergic and serotoninergic nervous systems. In addition, the increase in blood flow induced by TU-100 was antagonized by a CGRP antagonist, and partially antagonized by a vasoactive intestinal peptide antagonist and atropine. $^{2,6}$

In prior studies in humans, we previously showed that TU100 tended to accelerate small bowel and ascending colon transit in healthy volunteers, and in a study in 45 females with functional constipation without evidence of rectal evacuation disorder. ${ }^{7}$ Treatment for 28 days with TU-100, $5 \mathrm{~g}$ t.i.d. (means 3 times a day), was associated with trends of lower rectal sensation threshold for first sensation and gas compared to placebo, with no effects on psychosensory symptoms, stool frequency, stool consistency, or quality of life (QOL). Clinical experience with TU-100 in Japan for more than 20 years also suggests it is generally safe, and it reduces abdominal pain and bloating; however, the mechanism is unclear.

The current study hypothesis was that oral administration of TU-100 for a period of 14-16 days reduces rectal sensation in patients with irritable bowel syndrome (IBS) compared to placebo. The aim of this study was to assess the effects of orally administered TU 100, 5 g t.i.d., on rectal sensation, compliance, motility and symptoms in patients with IBS compared to placebo.

\section{Materials and Methods}

\section{Study Design}

This was a single-center, randomized, parallel-group, doubleblind, placebo controlled, single-response, pharmacodynamic study evaluating the effects of TU-100 on rectal sensation ratings and thresholds, compliance, bowel function and symptoms in patients with IBS. The protocol was approved by the Mayo Clinic Institutional Review Board.

A total daily dose of $15 \mathrm{~g}$ of TU-100 or matching placebo was administered orally for a minimum of 14 consecutive days (to 16 days to accommodate for final day coinciding with a weekend day). In a prior study, we evaluated the effects of TU-100 administered for 28 days $^{7}$; in this study, we used the shorter treatment period. There are no clinical trials that attempted to determine the pharmacokinetics or metabolism of TU-100. One clinical study examined the pharmacodynamic effects of TU-100. In 8 healthy adults, $5 \mathrm{~g}$ of TU-100 was administered into the stomach or duodenum through a manometric catheter and the GI motility was determined by GI manometry. An intense contractile response was observed at the administration site (stomach or duodenum), suggesting that TU-100 has a direct action on motility that commences soon after administration. ${ }^{8}$ TU-100 also increased blood levels of motilin and vasoactive intestinal polypeptide in humans within 3 hours of administration. ${ }^{9}$ Marketing studies from TU-100 use in Japan suggest onset of action is within hours or days. Therefore, there is rapid onset of effects of the medication, that is, within the 14 days of treatment in the current study, the medication's effects should be demonstrable.

\section{Randomization and Allocation}

Cato Research, a contract research organization (CRO), was responsible for providing a randomization schedule of medication doses randomly assigned in fixed block sizes to the Mayo Research Pharmacy in a format specified by the Mayo Pharmacy. The investigational pharmacy maintained the randomization schedule and only pharmacy personnel were allowed to see the randomization book and to know the block size, to avoid the possibility of prediction by the investigators. Thus, randomization and allocation were concealed from the study investigators.

\section{Study Population}

Participants were recruited from the local community by public advertisement or a targeted mailing. Forty-four female patients with 
IBS were screened and 40 were randomized. Twenty patients with IBS in each of the two treatment groups completed the study. Patients were screened by means of a short questionnaire and the IBSQOL questionnaire to assess GI symptoms and the impact on their QOL.

\section{Eligibility Criteria}

Patients fulfilled Rome III criteria for IBS. Females of childbearing potential were required to be using acceptable forms of contraception during the study and for 30 days after the last dose. The permitted age range was 18 to 65 years, inclusive, and permitted body mass index (BMI) was between 18 and $40 \mathrm{~kg} / \mathrm{m}^{2}$, inclusive. Patients were required to have negative urine drug screen at screen, no clinically significant laboratory results, normal rectal exam result on file within the past 2 years or performed at screen visit to exclude an evacuation disorder. Patients agreed to avoid alcohol during the entire study to avoid corrupting the data from the rectal studies.

Exclusion criteria were structural or metabolic diseases/conditions that affect the GI system (current or documented in the medical record, including clinical lactose intolerance), abnormal screening laboratory tests, taking any medication that had a potential to alter GI transit, alcoholics not in remission or known substance abusers, and history of allergic reactions to egg, ginseng, ginger or Sichuan pepper.

\section{Permitted Concurrent Medications}

Tricyclic antidepressants were permissible at a dose of $<25 \mathrm{mg}$ daily, and selective serotonin reuptake inhibitor antidepressants were permissible at low, stable doses. Analgesics such as acetaminophen, ibuprofen, naproxen and aspirin were permissible, except during sensation studies to avoid corrupting the data from the rectal barostat tests.

\section{Rescue Medications}

Rescue medications during the study medication treatment period were communicated to study staff, required approval by the principal investigator, and were documented by the patient in the bowel pattern diary. Rescue medications were not permitted within 7 days of the rectal sensation studies to ensure data integrity.

\section{Bowel Pattern, Bloating, and Pain Diary}

Throughout the duration of the study, patients recorded their symptoms in a daily diary that included documentation of each stool passed and its consistency (using the Bristol stool form scale), ease of passage, and sense of incomplete evacuation. In addition, on each day, patients recorded average bloating severity, average pain severity and worst pain severity during the day (all on $100 \mathrm{~mm}$ visual analog scales [VAS]). A follow-up visit was conducted on day 7 to 10 following cessation of treatment (variation to accommodate for the weekend days), and diaries of the daily recording of symptoms during the post-treatment period were collected.

\section{Irritable Bowel Syndrome Quality of Life}

The IBS-QOL questionnaire ${ }^{10}$ was completed by each patient immediately before starting the study medication and at the last dose of study medication.

\section{Experimental Protocol Appraising Rectal Sensory and Motor Functions}

Figure 1 shows the experimental protocol.

\section{Rectal Compliance and Sensation Thresholds}

All patients received bowel preparation $(250 \mathrm{~mL}$ warm tap water enema or 1-2 Fleets enemas, self-administered at least 30 minutes before testing commenced) and had fasted overnight. A rectal catheter with a polyethylene bag attached (length $22 \mathrm{~cm}$ and capacity $600 \mathrm{~mL}$ ) was inserted into the rectum so that the middle of the balloon was located approximately $10 \mathrm{~cm}$ from the anal verge.

To decrease the effects of abdominal viscera on the balloon volume, the patients were placed in a semi-prone position and the foot end of the bed elevated 15 degrees. The intrarectal balloon was unfolded by inflation with $75 \mathrm{~mL}$ of air and complete deflation. After a 5 minute recovery period, the catheter was connected to a barostat (G\&J Electronics Inc, Toronto, Ontario, Canada) and an

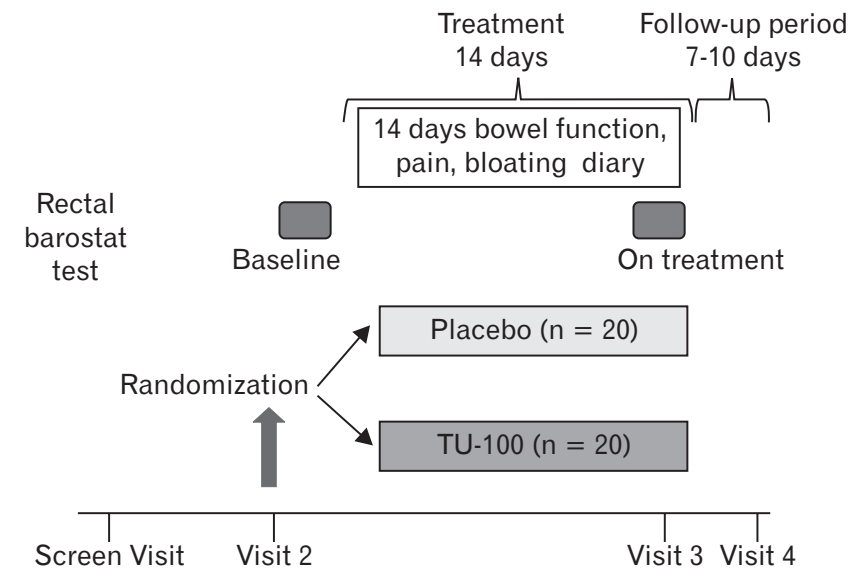

Figure 1. Experimental protocol: assessment of rectal compliance, sensation thresholds, ratings, and response to a meal. TU-100, Daikenchuto. 
initial "conditioning" distension of the rectum was then performed in which the pressure was increased from $0 \mathrm{mmHg}$ in steps of 4 $\mathrm{mmHg}$ for 15 seconds per step until $20 \mathrm{mmHg}$ was reached. This "conditioning" distension to $20 \mathrm{mmHg}$ rendered subsequent assessments of compliance and perception more reproducible. ${ }^{11}$ The bag was then deflated to $0 \mathrm{mmHg}$ and the patients were allowed to rest for 5 minutes before proceeding to the ascending method of limits. On the test day, all participants underwent baseline testing of compliance, sensation (thresholds and ratings) and baseline fasting tone, and then received study medication 1 hour prior to the ontreatment barostat testing which assessed rectal compliance, sensation thresholds, sensation ratings, fasting and postprandial tone (Fig. 1).

\section{Ascending Method of Limits: Compliance and Sensory Thresholds}

Rectal sensory thresholds and compliance were measured by ramp inflation, increasing in steps of $4 \mathrm{mmHg}$ for 1 minute per step from $0 \mathrm{mmHg}$ to a maximum $60 \mathrm{mmHg}$. Thresholds for sensations of gas, first sensation, urgency, and pain were indicated by the patients by pressing a button at the distension pressure at which the different sensations were perceived. Ramp inflation was terminated as soon as patients reported the first sensation of pain. ${ }^{12}$

\section{Rectal Sensation Ratings}

After the assessment of compliance and another 10-minute equilibration period, the operating pressure (that is, the pressure at which respiration induced fluctuations in recorded volume, indicating that the balloon was in apposition with the rectal wall) was set. Next, fasting rectal tone was measured at the operating pressure for a period of 10 minutes. Randomized-order phasic distensions were applied at 16, 24, 32, and $40 \mathrm{mmHg}$ above the operating pressure to measure the sensation ratings of urge to defecate, gas and pain. Each distention lasted 1 minute and was followed by an equilibration period at the operating pressure for 2 minutes.

A VAS was used to assess the level of anxiety or stress experienced by each subject, because these are potentially significant covariates in the assessment of visceral sensation scores.

The 100 mm VAS was anchored with the words "worried" and "relaxed" at the left and right ends of the scale; thus, a lower score reflects higher anxiety/stress levels.

\section{Rectal Motor Response to Feeding}

After the post-drug assessment of compliance and sensation, a baseline assessment of rectal tone was measured for 30 minutes with the rectal balloon at operating pressure. This was followed by a stan- dard liquid $1000 \mathrm{kcal}$ meal (750 mL chocolate milkshake, 53\% fat, $35 \%$ carbohydrate, and $12 \%$ protein) to measure the rectal response to feeding over 60 minutes. Thereafter, the balloon was deflated and the tube was removed.

\section{Endpoints}

The primary endpoints were:

(1) Sensation "rating" of urgency to defecate in response to 32 $\mathrm{mmHg}$ distension of the rectum on a $100 \mathrm{~mm}$ VAS.

(2) Sensation "threshold" for pain in response to distension of the rectum.

The secondary endpoints were:

(1) Physiological: rectal compliance $\left(\operatorname{Pr}_{1 / 2}\right.$, or pressure at half maximum volume), rectal sensation thresholds (gas, urgency to defecate, and first sensation), rectal sensation ratings (pain and gas), fasting rectal tone, rectal tone response to ingestion of a $1000 \mathrm{kcal}$ meal (measured as the delta barostat balloon volume and as symmetric percent change from fasting to postprandial balloon volume)

(2) Clinical: stool frequency (number per day) and consistency (on Bristol stool form scale), daily average severity of pain, worst severity of pain, and daily average bloating severity measured on $100 \mathrm{~mm}$ VAS.

(3) Quality of life score.

\section{Statistical Methods}

The study was analyzed in accordance with the intent-to-treat (ITT) principle, with all randomized patients included in the ITT analyses. Analyses based on this population were performed according to the randomized treatment. The safety population was defined as patients who took at least one dose of study drug, according to what patients actually received. Those patients withdrawing and/ or those with missing endpoint values for analysis had their missing data values imputed by using the overall (non-missing) mean value for the specific endpoint being analyzed. Specifically, the missing data were in sensation ratings at baseline or on treatment in response to distension at $16 \mathrm{mmHg}$ (1 patient), $24 \mathrm{mmHg}$ (3 patients), $32 \mathrm{mmHg}$ (2 patients), and $40 \mathrm{mmHg}$ (6 patients). Data were missing typically because of patient request to stop distension due to discomfort experienced at one-step lower distension level. An adjustment in the analysis of covariance (ANCOVA) degrees of freedom for error (subtracting one degree of freedom for each missing value imputed) was made to adjust the estimate of the residual error variance.

The overall effects of TU-100 treatment on the primary and 
some secondary endpoints were assessed by using an ANCOVA with rank transformation for skewness in the distributions of measured responses, if necessary. The covariates considered for inclusion in the analyses were baseline measurements and level of anxiety/stress in relation to sensations. $A$ priori anticipated contrasts (drug vs placebo) were examined $(\alpha=0.05)$.

Table 1 summarizes data for the primary response measures and uses the (relative) variation $(\mathrm{CV} \%)$ to estimate the effect size detectable with $80 \%$ power, based on a two-sample $t$ test at a twosided alpha level of 0.05 . The effect size is the difference in group means as a percentage of the overall mean for each response and assumes 20 patients per group. Based on data acquired in the laboratory, the sample size of 20 patients completed per group provides $80 \%$ power to detect approximately $40 \%$ effect sizes in the primary and important secondary pharmacodynamics endpoints of the study.

The frequency of adverse events was summarized by treatment group and by severity within treatment group, and was compared among treatment groups by using Fisher exact test. The frequency of all adverse events was compared among treatment groups by us- ing a Poisson regression model adjusting for age. All safety analyses were based on the safety population.

\section{Results}

\section{Patient Demographics and Clinical Endpoints}

Table 2 shows the demographics and baseline frequency and consistency of bowel movements of the 2 groups of 20 female patients; there were no differences in the 2 groups. Based on the symptoms reported in the baseline questionnaire, IBS subtype (diarrhea, alternators, and constipation) in each group (out of 20) were respectively 6,0 , and 14 in the placebo group and 5, 1, and 14 in the TU-100 group.

Table 2 also shows the effects of TU-100 or placebo treatment for a minimum of 14 days on clinical endpoints, including bowel function as well as scores of average daily bloating, average daily abdominal pain, and worst daily abdominal pain score in IBS. No significant treatment effects were observed. Table 2 also shows the post-treatment follow-up average daily symptom scores and

Table 1. Statistical Power Primary Response Measurement Summary

\begin{tabular}{|c|c|c|c|c|}
\hline \multicolumn{4}{|c|}{ Assuming $\mathrm{n}=20$ per group } & \multirow{2}{*}{$\begin{array}{c}\text { Effect size }^{\mathrm{a}}(\%) \\
\text { detectable with } 80 \% \text { power }(\alpha=0.05)\end{array}$} \\
\hline Response type & Mean & $\mathrm{SD}$ & $\mathrm{CV} \%$ & \\
\hline Rectal pain sensation ratings $32 \mathrm{mmHg}$ & 58 & 28 & 46 & 39 \\
\hline Rectal compliance $\left(\operatorname{Pr}_{1 / 2}\right) \mathrm{mmHg}(\mathrm{n}=47)$ & 12.5 & 5.4 & 43 & 40 \\
\hline Rectal sensation threshold $(n=47)$ & 29 & 14 & 48 & 45 \\
\hline
\end{tabular}

${ }^{a}$ Effect size is the difference between group means as a percentage of overall groups mean.

$\mathrm{CV}$, coefficient of variation; $\mathrm{Pr}_{1 / 2}$, pressure at half maximum volume where a lower $\operatorname{Pr}_{1 / 2}$ reflects higher compliance; $\mathrm{SD}$, standard deviation.

Table 2. Baseline Demographics and Effects of Daikenchuto Treatment for 10 Days on Clinical Endpoints in Irritable Bowel Syndrome, Including Follow-up Post-treatment (mean \pm SEM)

\begin{tabular}{|c|c|c|c|c|c|c|}
\hline Symptom & $\begin{array}{l}\text { Baseline } \\
\text { placebo }\end{array}$ & $\begin{array}{c}\text { Placebo } \\
\text { Rx period }\end{array}$ & $\begin{array}{l}\text { Placebo } \\
\text { follow-up }\end{array}$ & $\begin{array}{l}\text { Baseline } \\
\text { TU-100 }\end{array}$ & $\begin{array}{c}\text { TU-100 } \\
\text { Rx period }\end{array}$ & $\begin{array}{l}\text { TU-100 } \\
\text { follow-up }\end{array}$ \\
\hline Number & 20 & & & 20 & & \\
\hline Age (yr) & $43.0 \pm 2.6$ & & & $39.5 \pm 2.7$ & & \\
\hline $\operatorname{BMI}\left(\mathrm{kg} / \mathrm{m}^{2}\right)$ & $27.5 \pm 1.0$ & & & $28.5 \pm 1.6$ & & \\
\hline No. of stools/day & $1.62 \pm 0.16$ & $1.58 \pm 0.13$ & $1.64 \pm 0.16$ & $1.49 \pm 0.12$ & $1.51 \pm 0.08$ & $1.42 \pm 0.11$ \\
\hline Stool form BSFS (1-7) & $3.55 \pm 0.28$ & $3.66 \pm 0.23$ & $3.50 \pm 0.27$ & $3.55 \pm 0.31$ & $3.83 \pm 0.25$ & $3.48 \pm 0.30$ \\
\hline Bloating (100 mm VAS) & $26.0 \pm 4.18$ & $21.2 \pm 3.65$ & $26.6 \pm 4.90$ & $30.5 \pm 4.87$ & $23.9 \pm 3.95$ & $25.5 \pm 5.69$ \\
\hline Average pain (100 mm VAS) & $19.02 \pm 3.99$ & $16.12 \pm 3.25$ & $17.77 \pm 3.73$ & $21.09 \pm 4.99$ & $13.95 \pm 3.94$ & $14.12 \pm 4.41$ \\
\hline Worst pain (100 mm VAS) & $25.28 \pm 5.31$ & $20.38 \pm 3.93$ & $23.44 \pm 5.15$ & $27.27 \pm 5.43$ & $18.81 \pm 4.47$ & $19.52 \pm 5.60$ \\
\hline IBS-QOL & $0.17 \pm 0.02$ & $0.14 \pm 0.02$ & ND & $0.16 \pm 0.03$ & $0.14 \pm 0.03$ & ND \\
\hline
\end{tabular}

Rx, treatment; TU-100, Daikenchuto; BMI, body mass index; BSFS, Bristol stool form scale; VAS, visual analog scale; IBS, irritable bowel syndrome; QOL, quality of life; ND, not determined. 

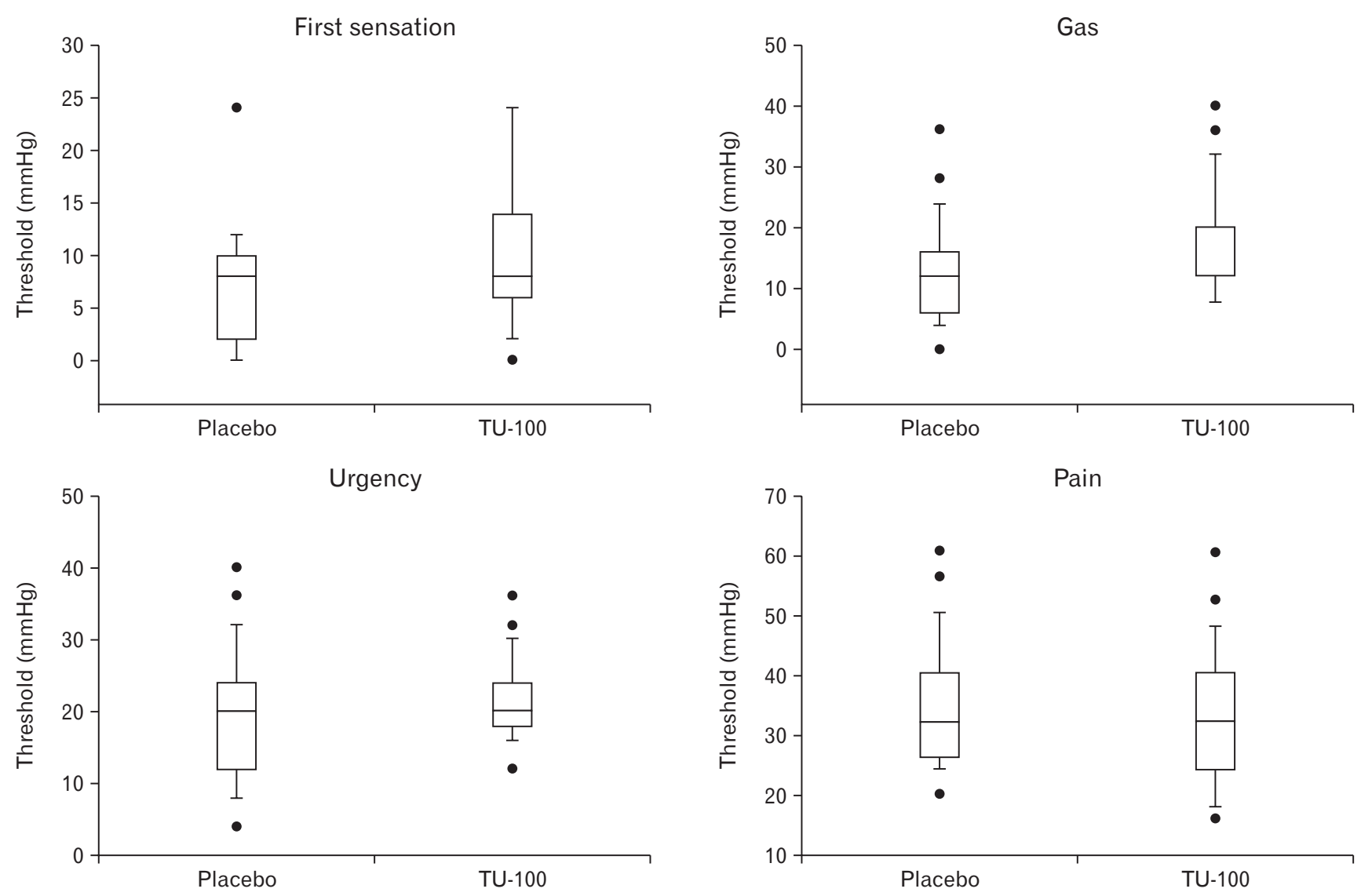

Figure 2. Effects of Daikenchuto (TU-100) and placebo on rectal sensation thresholds.

illustrates no numerical differences relative to the baseline or ontreatment periods.

\section{Rectal Sensation Ratings and Thresholds}

Figure 2 shows the rectal sensation thresholds (first sensation, gas, urge to defecate, and pain). Simultaneously with thresholds, rectal compliance $\left(\operatorname{Pr}_{1 / 2}\right)$ was measured (Fig. 3). There were no significant differences between the 2 treatment groups.

Rectal sensation ratings in response to treatment were significantly associated with baseline (pre-treatment) ratings (Table 3) and at some pressures, with level of anxiety or stress recorded at the time of the sensation testing (Table 4, showing Spearman correlation coefficients). Thus, there was an association of anxiety with sensations of urge and pain at low level of distension pressure $(16 \mathrm{mmHg})$; the association with anxiety and stress was not consistently identified at higher levels of pressure distension. The negative correlation coefficients reflect the fact that lower score on "worried to relaxed" reflects higher anxiety/stress level.

On the other hand, there were no effects of TU-100 treatment

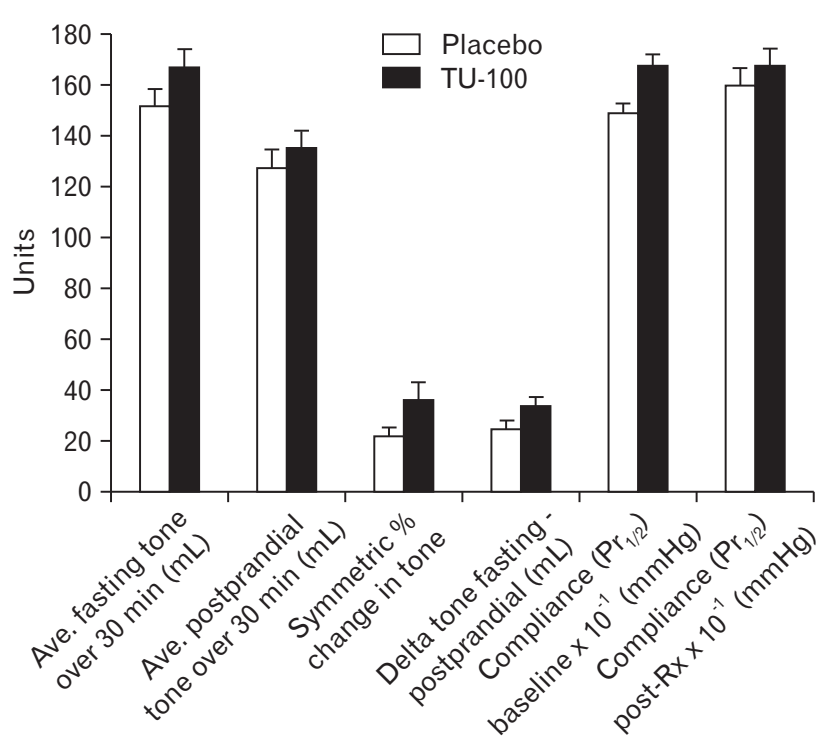

Figure 3. Effects of Daikenchuto (TU-100) on fasting and postprandial rectal tone. $\mathrm{Rx}$, treatment. 
Table 3. Significant Association of Baseline and Post-treatment Sensation Ratings in Response to Different Pressure Levels of Rectal Distension in Entire Study Cohort $(\mathrm{N}=40)$

\begin{tabular}{|c|c|c|c|c|c|c|c|c|c|c|c|c|}
\hline \multirow{2}{*}{$\begin{array}{l}\text { Sensation } \\
\text { Pressure }\end{array}$} & \multicolumn{4}{|c|}{ Gas } & \multicolumn{4}{|c|}{ Urge } & \multicolumn{4}{|c|}{ Pain } \\
\hline & Baseline & Post-Rx & Rs & $P$-value & Baseline & Post-Rx & Rs & $P$-value & Baseline & Post-Rx & Rs & $P$-value \\
\hline $16 \mathrm{mmHg}$ & $50.3 \pm 4.1$ & $43.7 \pm 3.5$ & 0.43 & 0.007 & $53.3 \pm 3.4$ & $44.6 \pm 4.1$ & 0.33 & 0.040 & $43.2 \pm 3.9$ & $33.7 \pm 3.8$ & 0.36 & 0.030 \\
\hline $24 \mathrm{mmHg}$ & $56.2 \pm 3.8$ & $52.8 \pm 3.8$ & 0.35 & 0.040 & $68.2 \pm 3.4$ & $57.0 \pm 4.2$ & 0.61 & $<0.001$ & $51.4 \pm 4.5$ & $47.3 \pm 4.4$ & 0.56 & $<0.001$ \\
\hline $32 \mathrm{mmHg}$ & $55.5 \pm 4.9$ & $64.4 \pm 3.3$ & 0.53 & $<0.001$ & $57.5 \pm 3.8$ & $70.7 \pm 3.6$ & 0.45 & 0.006 & $55.6 \pm 4.4$ & $60.5 \pm 4.6$ & 0.40 & 0.016 \\
\hline $40 \mathrm{mmHg}$ & $60.3 \pm 5.2$ & $67.2 \pm 4.0$ & 0.49 & 0.005 & $77.8 \pm 3.1$ & $73.7 \pm 3.3$ & 0.20 & 0.270 & $66.3 \pm 4.8$ & $68.2 \pm 3.8$ & 0.50 & 0.004 \\
\hline
\end{tabular}

$\mathrm{Rx}$, treatment; Rs, Spearman correlation coefficient.

Table 4. Spearman Correlation Coefficients Between Anxiety/Stress Ratings During Sensation Tests on Treatment and Sensation Ratings for Gas, Urge, and Pain

\begin{tabular}{|c|c|c|c|c|c|c|}
\hline \multirow{2}{*}{$\begin{array}{c}\text { Sensation } \\
\text { Pressure }\end{array}$} & \multicolumn{2}{|c|}{ Gas } & \multicolumn{2}{|c|}{ Urge } & \multicolumn{2}{|c|}{ Pain } \\
\hline & Rs & $P$-value & Rs & $P$-value & Rs & $P$-value \\
\hline $16 \mathrm{mmHg}$ & -0.26 & 0.110 & -0.47 & 0.006 & -0.55 & $<0.001$ \\
\hline $24 \mathrm{mmHg}$ & 0.11 & 0.500 & -0.17 & 0.304 & -0.18 & 0.290 \\
\hline $32 \mathrm{mmHg}$ & 0.02 & 0.920 & -0.05 & 0.770 & -0.06 & 0.730 \\
\hline $40 \mathrm{mmHg}$ & 0.03 & 0.880 & -0.13 & 0.440 & -0.33 & 0.054 \\
\hline
\end{tabular}

Rs, Spearman correlation coefficient.

Note the association of anxiety with sensations of urge and pain at low level of distension pressure $(16 \mathrm{mmHg})$.

on rectal sensation ratings of gas, urge to defecate, or pain compared to placebo (Fig. 4) in response to the 4 levels of pressure distension, including the pre-specified primary endpoint, $32 \mathrm{mmHg}$ distension.

\section{Fasting and Postprandial Rectal Tone}

Figure 3 shows the effects of treatment on fasting and postprandial rectal tone, expressed as the change in rectal balloon volume, as well as the symmetric percent change in rectal balloon volume measured with the barostat.

\section{Discussion}

This randomized, controlled trial appraised the pharmacodynamics effects of a single dose of TU-100 on rectal sensation ratings in female patients with IBS. Post-treatment sensation ratings were significantly associated with baseline (pre-treatment) ratings and with level of anxiety or stress recorded at the time of the sensation testing.

In addition, Table 2 shows that, in the follow-up period, the reduction in sensation ratings (VAS for bloating, average pain, and worst pain) observed in both groups after treatment was numerically larger (though not significant) in the TU-100 group. Thus, whereas the reduced scores on treatment in the placebo group returned to the original baseline values, we did observe continued decline in sensation ratings in the TU-100 group.

Overall, there were no effects of TU-100 treatment on rectal sensation ratings, sensation thresholds, rectal fasting or postprandial tone, rectal compliance, bowel function, abdominal pain or bloating scores, or IBS-QOL. These results confirm our previous observations that TU-100 does not significantly alter rectal compliance or rectal sensation thresholds in patients with functional constipation, although there were borderline effects on first sensation and gas (unadjusted $P$-values: 0.045 and 0.024 ) with the 5 g t.i.d. dose, ${ }^{7}$ which was the dose tested in the current study.

These data suggest that the beneficial clinical effects of TU100 reported in Japanese patients with postoperative ileus or adhesive obstruction may be related more closely to the motor effects of the medication or to changes in colonic microbiota that were not established with 2 weeks of treatment in our clinical trial. Other studies have demonstrated important anti-inflammatory effects of TU-100 on inflammatory mediators; TU-100 suppresses TNF- $\alpha$ and IFN- $\gamma$ activity in the intestinal mucosa of Crohn's disease by inducing endogenous adrenomedullin with anti-TNF- $\alpha$ and antiIFN- $\gamma$ activity. ${ }^{13}$

This study also failed to show any significant effects on clinical 
Gas ratings
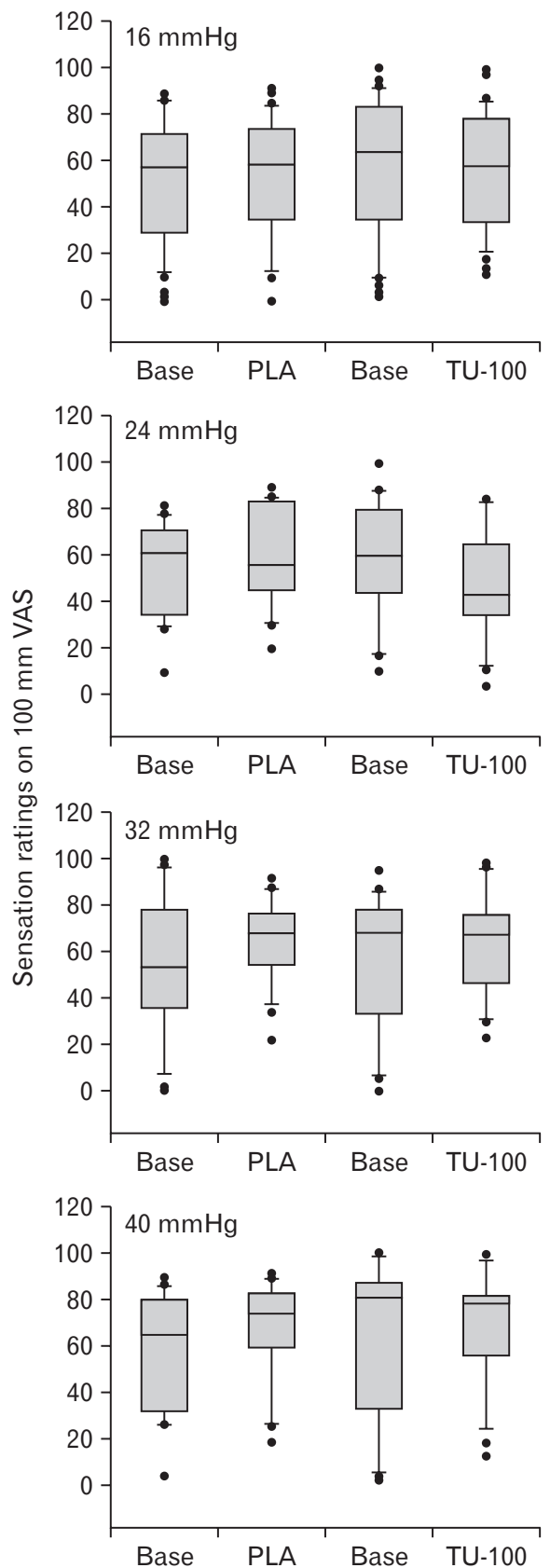

Urge to defecate ratings
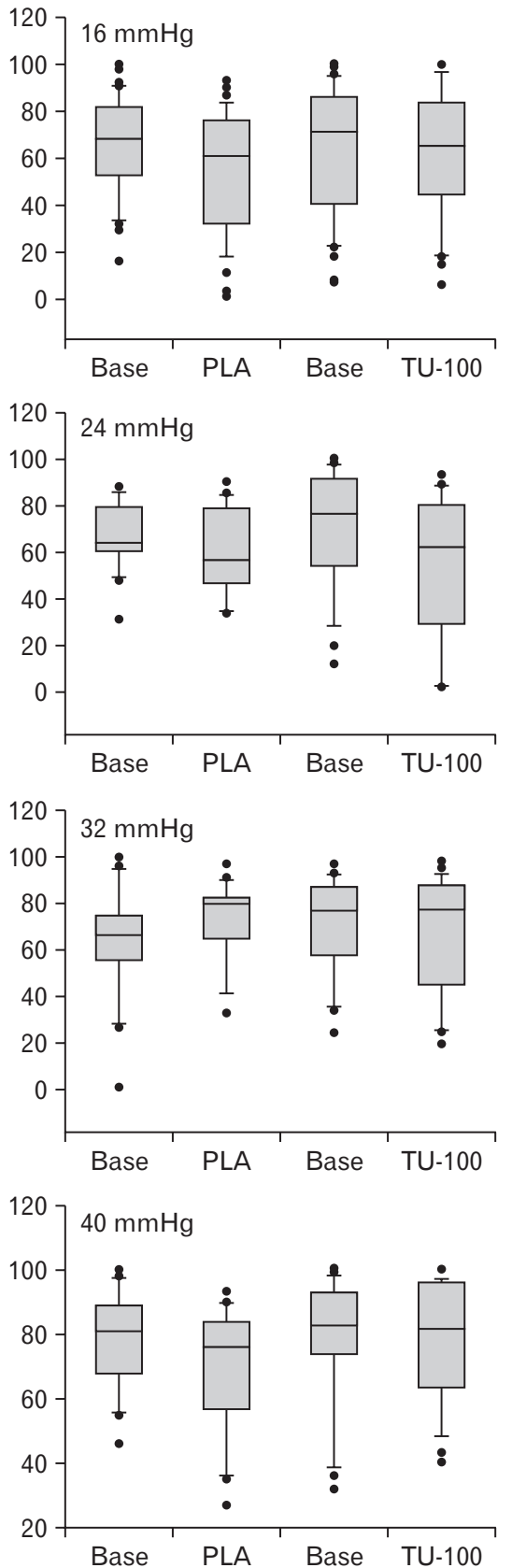

Pain ratings
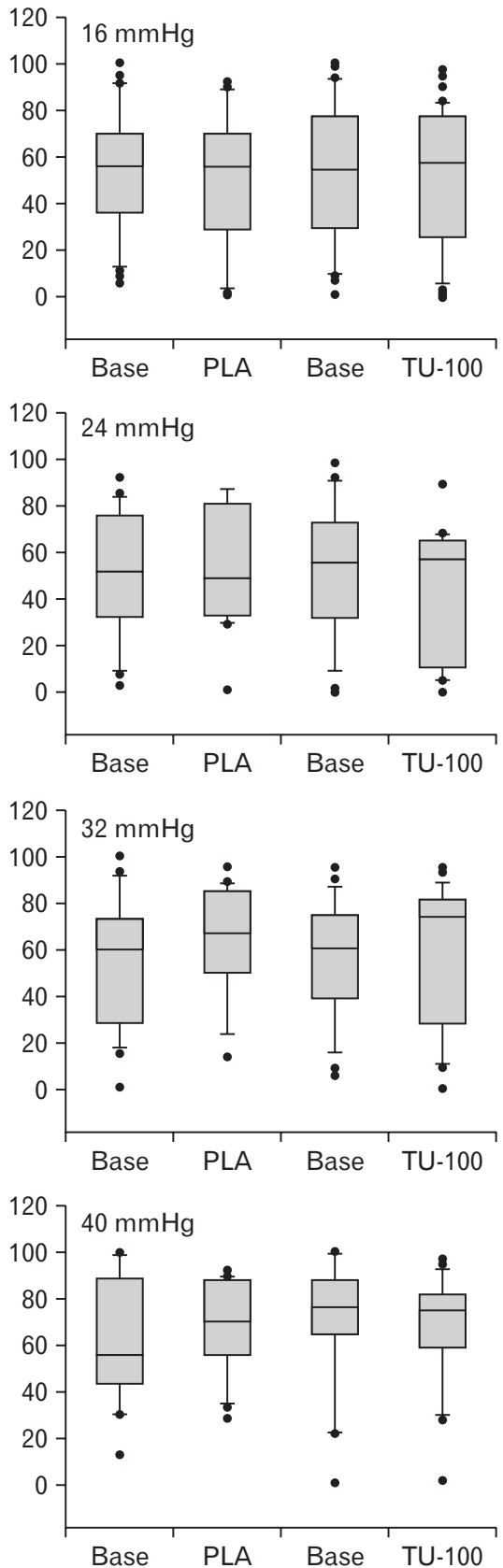

Figure 4. Effects of Daikenchuto (TU-100) and placebo (PLA) on sensation ratings of gas, urge to defecate and pain during rectal distensions at $16,24,32$, and $40 \mathrm{mmHg}$ pressure. VAS, visual analog scale.

endpoints and IBS-QOL during a 14-day treatment period. However, the study was of short duration, evaluated a single dose, and had a small sample size to appraise the clinical efficacy in IBS. In order to appraise the clinical efficacy, a randomized, controlled trial is currently testing the safety and efficacy of TU-100 to relieve abdominal bloating in females with IBS (ClinicalTrials.gov Identifier:
NCT02074579). Our study differs from the findings in patients with functional constipation ${ }^{7}$ and it is conceivable that the degree of baseline hypersensitivity may have impacted the differences in outcomes in the 2 studies. Another potential explanation for the differences in efficacy observed in clinical practice in Japanese patients and the lack of pharmacodynamic parameters of sensation 
observed in patients in America may reflect the previously observed differences in pharmacokinetics between Japanese and US healthy volunteers. ${ }^{14}$ Factors that may account for these differences were suggested from simulation studies and these included differences in BMI, body weight and age.

In summary, TU-100 does not significantly affect rectal function in patients with IBS. Further studies are needed to evaluate its clinical efficacy and safety in patients with IBS.

Financial support: This study was supported by a research grant and study medication from Tsumura USA Inc.

\section{Conflicts of interest: None.}

Author contributions: Andres Acosta: analysis and interpretation of data, conduct of the study, and writing and critical revision of the manuscript; Michael Camilleri: study concept and design, analysis and interpretation of data, drafting of the manuscript, and critical revision of the manuscript; Sara Linker Nord: conduct of sensation studies; Irene Busciglio: conduct of sensation studies; Johanna Iturrino: eligibility of participant and conduct of the study; Lawrence A Szarka: conduct of the study; and Alan R Zinsmeister: study design, analysis and interpretation of data, and critical revision of the manuscript.

\section{ClinicalTrials.gov identifier: NCT01890837.}

\section{References}

1. Takeda T, Kamiura S, Kimura T. Effectiveness of the herbal medicine daikenchuto for radiation-induced enteritis. J Altern Complement Med 2008;14:753-755.

2. Kono T, Koseki T, Chiba S, et al. Colonic vascular conductance increased by Daikenchuto via calcitonin gene-related peptide and receptor-activity modifying protein 1. J Surg Res 2008;150:78-84

3. Christianson JA, Bielefeldt K, Altier C, et al. Development, plasticity and modulation of visceral afferents. Brain Res Rev 2009;60:171-186.

4. Shibata C, Sasaki I, Naito H, Ueno T, Matsuno S. The herbal medicine Dai Kenchu-Tou stimulates upper gut motility through cholinergic and 5 hydroxytryptamine 3 receptors in conscious dogs. Surgery 1999;126:918924.

5. Jin XL, Shibata C, Naito H, et al. Intraduodenal and intrajejunal administration of the herbal medicine, dai kenchu-tou, stimulates small intestinal motility via cholinergic receptors in conscious dogs. Dig Dis Sci 2001;46: 1171-1176.

6. Murata P, Hayakawa T, Satoh K, Kase Y, Ishige A, Sasaki H. Effects of Dai kenchu-to, a herbal medicine, on uterine and intestinal motility. Phytother Res 2001;15:302-306.

7. Iturrino J, Camilleri M, Wong BS, Linker Nord SJ, Burton D, Zinsmeister AR. Randomised clinical trial: the effects of daikenchuto, TU100 , on gastrointestinal and colonic transit, anorectal and bowel function in female patients with functional constipation. Aliment Pharmacol Ther 2013;37:776-785

8. Furukawa Y, Shiga Y, Hanyu N, et al. [Effect of Chinese herbal medicine on gastronintestinal motility and bowel obstruction.] Jpn J Gastroenterol Surg 1995;28:956-960. [Japanese]

9. Sato Y, Inoue S, Katagiri F, Itoh H, Takeyama M. Effects of pirenzepine on Dai-kenchu-to-induced elevation of the plasma neuropeptide levels in humans. Biol Pharm Bull 2006;29:166-171.

10. Patrick DL, Drossman DA, Frederick IO, DiCesare J, Puder KL. Quality of life in persons with irritable bowel syndrome: development and validation of a new measure. Dig Dis Sci 1998;43:400-411.

11. Hammer HF, Phillips SF, Camilleri M, Hanson RB. Rectal tone, distensibility, and perception: reproducibility and response to different distensions. Am J Physiol 1998;274(3 Pt 1):G584-G590.

12. Camilleri M, McKinzie S, Busciglio I, et al. Prospective study of motor, sensory, psychologic, and autonomic functions in patients with irritable bowel syndrome. Clin Gastroenterol Hepatol 2008;6:772-781.

13. Ueno N, Musch M, Hasebe T, et al. Multi-targeted treatment of experimental colitis by daikenchuto, a traditional Japanese herbal medicine. In: Proceedings of The Physiological Society, 37th Congress of IUPS. Birmingham, UK, 2013.

14. Munekage M, Ichikawa K, Kitagawa H, et al. Population pharmacokinetic analysis of daikenchuto, a traditional Japanese medicine (Kampo) in Japanese and US health volunteers. Drug Metab Dispos 2013;41:12561263. 